higher risk for reinfections were young people ( $<20$ years $17 \%$ ), specific ethnic minorities (Netherlands Antillean 16\%, Turkish 17\%, sub-Sahara African 18\%), persons living in Rotterdam (11 vs $8 \%$ Amsterdam 4\% Limburg), and in high-risk areas (14\%).

Conclusions The uptake of retesting was successful counting two third with automatically sent testkits 6 months after screening. Reinfection rates were high, especially among known risk-groups. Questionnaire results show that follow-up of (partner) treatment after Chlamydia infections could be improved.

\section{1-S01.04 SUBOPTIMAL REPEAT TESTING OF WOMEN WITH POSITIVE CHLAMYDIA TESTS IN THE USA, 2008-2010}

doi:10.1136/sextrans-2011-050109.4

${ }^{1} \mathrm{~K}$ Hoover, ${ }^{1} \mathrm{G}$ Tao, ${ }^{2} \mathrm{~B}$ Body, ${ }^{2} \mathrm{M}$ Nye, ${ }^{1} \mathrm{C}$ Kent. ${ }^{1} \mathrm{CDC}$, Atlanta, USA; ${ }^{2}$ Laboratory Corporation of America, USA

Background Women treated for chlamydia have a high prevalence of infection several months later, likely caused by reinfection from an untreated or new infected sex partner. To prevent potential adverse outcomes of chlamydia, US guidelines recommend repeat testing 3 months after treatment, regardless of partner treatment. If retesting at 3 months is not possible, women should be retested at their next clinical encounter within 12 months. A chlamydia test-ofcure is also recommended for all infected pregnant women 3-4 weeks after treatment. We assessed adherence to retesting guidelines using data from a US laboratory corporation that has a large share of the US market.

Methods Among tests performed from June 2008 to May 2010, we estimated the percentage of women who were retested $\geq 3$ weeks later by test result, age and pregnancy status. We also estimated the positivity rate among repeat chlamydia tests and the mean time between an initial test and the first repeat test. We assumed that for each woman in the database all chlamydia tests during the study period were performed by this laboratory corporation.

Results Among 2.90 million chlamydia tests performed in 1.77 million women, 4.0\% (114963) were positive. Among the 1.77 million women with tests, 1.34 million $(75.7 \%)$ had only a single test and 0.43 million ( $24.3 \%$ ) had at least one repeat test. If an initial test was positive, $48.6 \%$ were retested compared to $23.5 \%$ if the initial test was negative $(p<0.01)$; a repeat test was more likely to be positive in women with an initial positive test $(13.3 \%)$ than a negative one $(3.3 \%)(p<0.01)$. The mean time interval between the initial and repeat test was shorter if the initial test was positive (117 days) than negative (149 days). Women aged 15-24 years with a positive test had a lower retesting rate than those aged $25-34$ years (46.8\% vs $53.3 \%$ ). The percentage of women with a positive test who were retested differed significantly by pregnancy status $(60.0 \%$ pregnant vs $44.2 \%$ nonpregnant), and pregnant women had a repeat test within 93 days compared to 125 days in nonpregnant women. Conclusions These data from a large laboratory corporation provide insight into chlamydia testing practices among women in the USA, and suggest suboptimal adherence to retesting recommendations for both pregnant and nonpregnant women. These data can be useful to monitor the effectiveness of interventions to improve follow-up testing of women with chlamydia.

\section{1-S01.05 ESTIMATING THE RATE OF ANNUAL CHLAMYDIA SCREENING UPTAKE IN US WOMEN}

doi:10.1136/sextrans-2011-050109.5

${ }^{1} \mathrm{~J}$ Heijne, ${ }^{1} \mathrm{C}$ Althaus, ${ }^{1} \mathrm{~S}$ Herzog, ${ }^{2} \mathrm{G}$ Tao, ${ }^{2} \mathrm{C}$ Kent, ${ }^{1} \mathrm{~N}$ Low. ${ }^{1}$ University of Bern, Bern, Switzerland; ${ }^{2}$ Centers for Disease Control \& Prevention (CDC), Atlanta, USA

Background Annual chlamydia testing of all sexually active women aged 25 years and under followed by a repeated test 3 months after treatment is recommended by the US Centers for Disease Control and Prevention (CDC) guidelines. Data from the Healthcare Effectiveness Data and Information Set (HEDIS) estimate chlamydia test coverage at about $40 \%$ per year in the last 5 years. We used empirical data and mathematical models to determine whether observed patterns of chlamydia testing are consistent with CDC guidelines and with actual trends in chlamydia prevalence in US women aged 15-25 years.

Methods First, published data from women enrolled in commercial health plans from 2002 to $2006(n=1985920)$ were used to estimate the annual chlamydia testing rate in women aged 15-25. Second, trends in chlamydia prevalence in the same age group were studied using data from 1999/2000 to 2007/2008 (n 600 each round) from the US National Health and Nutrition Examination Survey (NHANES). We used a Susceptible-Infected-Recovered-Susceptible (SIRS) model to estimate the annual screening rate that fit the chlamydia prevalence data best. The model described a closed population with behavioural parameters reflecting people aged 15-25 years. It explicitly incorporated sexual partnerships and took into account re-infection. Finally, the model was used to examine the effect of repeated chlamydia testing 3 months after treatment on chlamydia prevalence and to calculate repeat infection rates.

Results The estimated rate at which women are tested for chlamydia ranges from 0.06 to 0.11 per year, which corresponds to a chlamydia test every 9 to 16 years on average and an annual coverage of roughly $10 \%$. We found no statistical evidence that chlamydia prevalence changed between 1999 and 2008 in sexually active women aged $15-25$ years taking part in NHANES. Predictions from the model of the impact of screening at a rate of 0.11 per year were consistent with the observed stable chlamydia prevalence. Repeat chlamydia testing 3 months after treatment at the estimated screening level hardly influenced population prevalence. The percentage of women with a repeat infection was highest 3.8 months after treatment.

Conclusion Our study demonstrates the challenges of implementing chlamydia screening. This study suggests that low rates of chlamydia testing in the US have not reduced population chlamydia prevalence substantially.

\section{1-S01.06 ESTIMATION OF THE BURDEN OF DISEASE AND COSTS OF GENITAL CHLAMYDIA TRACHOMATIS INFECTION IN CANADA}

doi:10.1136/sextrans-2011-050109.6

A Tuite, D Fisman. University of Toronto, Toronto, Canada

Background Chlamydia trachomatis (CT) is the most common notifiable infectious disease in Canada. Rates of infection have been increasing since the mid-1990s, suggesting that alternate control strategies may be required. Given that the current cost of Chlamydia infections in Canada is unknown, we sought to estimate the burden of CT in the Canadian population, to provide a foundation on which health economic analyses of competing Chlamydia control strategies can be constructed.

Methods We used an age- and sex-structured mathematical model parameterised to reproduce trends in CT prevalence in the Canadian population aged 10 to 39 years. Model parameters were derived from epidemiologic studies and by model calibration. We incorporated data on changing test patterns of asymptomatically infected individuals over time. Costs were identified, measured, and valued using a modified societal perspective and were converted to 2009 Canadian dollars. The main outcome measures were the current net cost and burden of illness attributable to CT infection.

Results The model reproduced trends in CT prevalence observed for the time period between 1991 and 2008. Under base case model assumptions, there appeared to be a trend of increasing detection of 
CT cases (due to increases in screening and subsequent detection of asymptomatic cases), despite an underlying decrease in actual CT infections in the population. The total estimated costs associated with CT infection over this time period were over $\$ 1.0$ billion, or $\$ 56.4$ million per year. The costs of screening and treatment of asymptomatic infections as a proportion of total CT costs were estimated to have increased over time, while the costs of long-term sequelae associated with untreated infections declined the same period.

Conclusions Despite increases in screening over time, the total economic burden associated with CT in Canada remains high; however, the projections of our model suggest that these increases in screening and the subsequent detection of asymptomatic infections may be reducing the costs associated with the treatment downstream sequelae of untreated infections. Further investigation of trends in chlamydia-associated complications is required to better understand the impact of screening on CT incidence in Canada.

\section{Epidemiology oral session 2: Human papillomavirus}

\section{1-S02.01 EPIDEMIOLOGY OF, AND BEHAVIOURAL RISK FACTORS FOR, SEXUALLY TRANSMITTED HUMAN PAPILLOMA VIRUS INFECTION IN A SAMPLE OF THE BRITISH POPULATION}

doi:10.1136/sextrans-2011-050109.7

${ }^{1} \mathrm{C}$ H Mercer, ${ }^{1} \mathrm{~A}$ M Johnson, ${ }^{2} \mathrm{~N}$ de Silva, ${ }^{2} \mathrm{~S}$ Beddows, ${ }^{2} \mathrm{~S}$ Desai, ${ }^{2} \mathrm{R}$ Howell-Jones, ${ }^{3} \mathrm{C}$ Carder, ${ }^{1} \mathrm{P}$ Sonnenberg, ${ }^{2} \mathrm{C} \mathrm{M}$ Lowndes, ${ }^{2} \mathrm{~K}$ Soldan. ${ }^{1}$ University College London, London, UK; ${ }^{2}$ Health Protection Agency, London, UK; ${ }^{3}$ University College Hospitals NHS Foundation Trust, UK

Background Persistent infection with high-risk sexually transmitted human papilloma viruses (HR-HPV) can lead to development of cervical and other cancers while low-risk types (LR-HPV) may cause genital warts, the most commonly-diagnosed viral STI in the UK. An HPV immunisation programme, using the bivalent vaccine protecting against types 16 and 18, was introduced in the UK in 2008. The frequency of HPV types is important baseline information against which to monitor the direct and indirect effects of vaccination. Here we examine the proportion of the population with detectable infection with HPV in urine collected in 1999-2001 for the National Survey of Sexual Attitudes and Lifestyles (Natsal-2) and the relationship with demographic and behavioural variables.
Methods Natsal-2 was a probability sample survey of men and women aged 16-44 resident in Britain involving computer-assisted personal interviewing. Half of all sexually-experienced respondents aged 18-44 were invited to provide a urine sample. 3436 samples were tested using an in-house Luminex-based HPV genotyping system. Results HPV DNA was detected in $29.0 \%$ (95\% CI $26.7 \%$ to $31.3 \%$ ) of samples from women and $17.4 \%$ (95\% CI $15.1 \%$ to $19.8 \%$ ) from men. Any of $13 \mathrm{HR}-\mathrm{HPV}$ types was detected in $15.9 \%(95 \% \mathrm{CI}$ $14.1 \%$ to $17.8 \%$ ) of women's samples and $9.6 \%$ (95\% CI $8.0 \%$ to $11.6 \%$ ) of men's. Vaccine preventable types 16 and/or 18 were found in $5.5 \%(95 \%$ CI $4.5 \%$ to $6.8 \%)$ of women and $3.0 \%$ (95\% CI $2.1 \%$ to $4.3 \%$ ) of men; and types 6 and/or 11 in $4.7 \%$ (95\% CI $1.8 \%$ to $3.3 \%)$ of women and $2.2 \%$ (95\% CI $1.5 \%$ to $3.1 \%)$ of men. $4.1 \%(95 \% \mathrm{CI}$ $3.1 \%$ to $5.2 \%$ ) of women had HPV 16 and/or 18 without any other HR-HPV. In multivariate analysis, HR-HPV was associated with number of new partners, in women with younger age, single status, and partner concurrency, and in men with number of unprotected partnerships and age at first intercourse.

Conclusion This is the first population-based probability sample study of the distribution of sexually transmissible HPV types in Britain. It is also the first to undertake a detailed analysis of relationships with demographic and behavioural variables and to include men. HPV DNA was detectable in urine of a high proportion of the sexually active British population; the lower prevalence in males reflected lower detection sensitivity for HPV in urine from males. In both genders HPV was strongly associated with sexual risk behaviour.

\section{1-S02.02 ARE THERE MUTUAL ASSOCIATIONS BETWEEN THE INCIDENCE OF HPV INFECTION AND OTHER SEXUALLY TRANSMITTED INFECTIONS AFTER CONTROLLING FOR SEXUAL BEHAVIOUR?}

doi:10.1136/sextrans-2011-050109.8

${ }^{1}$ Thing Rinda Soong, ${ }^{1} \mathrm{P}$ E Gravitt, ${ }^{2} \mathrm{~S}$ B Gupta, ${ }^{2} \mathrm{~A}$ Tadesse, ${ }^{2} \mathrm{E}$ Kim, ${ }^{3} \mathrm{C}$ Phongnarisorn, ${ }^{4} \mathrm{~V}$ Wootipoom, ${ }^{5} \mathrm{P}$ Yuenyao, ${ }^{1} \mathrm{D} D$ Celentano. ${ }^{1}$ Johns Hopkins Bloomberg School of Public Health, Baltimore, USA; ${ }^{2}$ Merck \& Co. Inc., USA; ${ }^{3}$ Chiang Mai University, Thailand; ${ }^{4}$ Prince of Songkla University, HadYai, Thailand; ${ }^{5}$ Khon Kaen University, Thailand

Background We aimed to determine (i) if other sexually transmitted infections (STIs) increase the risk of incident human papillomavirus (HPV) infection and (ii) if HPV infection predicts the incidence of other STIs.

Abstract 01-S02.02 Table 1 Unadjusted and adjusted estimates of detection of other STIs on HPV incidence (total number of visit pairs=3221)

\begin{tabular}{|c|c|c|c|c|}
\hline & $\begin{array}{l}\text { Number of visit } \\
\text { pairs } N=3221\end{array}$ & $\begin{array}{l}\text { Incident detection } \\
\text { at current visit }\end{array}$ & Unadjusted $\mathrm{OR} \dagger(95 \% \mathrm{Cl})$ & Adjusted $\mathrm{OR} \dagger(95 \% \mathrm{CI})$ \\
\hline Diagnosis of the following at previous visit & $N(\mathrm{col} \%)$ & $\begin{array}{l}\text { New cases of any HPV, } \\
n=241(7.5 \%) n \text { (row\%) }\end{array}$ & \multicolumn{2}{|c|}{ New detection of any HPV type across consecutive visits } \\
\hline \multicolumn{5}{|l|}{ STIs other than HPV infection* } \\
\hline No & $3158(98.0)$ & $230(7.3)$ & 1.0 & 1.0 \\
\hline \multirow[t]{2}{*}{ Yes } & $63(2.0)$ & $11(17.5)$ & $2.46(1.31$ to 4.62$)$ & $2.16(1.08$ to 4.34$)$ \\
\hline & & $\begin{array}{l}\text { New cases of any HR- } \\
\text { HPV }, n=110 \text { (3.4\%) } \\
n(\text { row } \%)\end{array}$ & \multicolumn{2}{|c|}{ New detection of any HR- HPV type across consecutive visits } \\
\hline \multicolumn{5}{|l|}{ STIs other than HPV infection* } \\
\hline No & $3158(98.0)$ & $105(3.3)$ & 1.0 & 1.0 \\
\hline Yes & $63(2.0)$ & $5(7.9)$ & $2.42(0.93$ to 6.27$)$ & $2.01(0.74$ to 5.48$)$ \\
\hline
\end{tabular}

*STIs other than HPV infection included the following: laboratory diagnoses of genital chlamydia, gonorrhoea, syphilis, as well as clinical diagnoses of genital herpes or trichomoniasis. †Estimates adjusted for age and study site at enrolment, as well as the following covariates assessed at each follow-up visit: pap smear diagnosis at previous visit, contraceptive use in last 6 months, number of lifetime partners, partners having sex with others in last 6 months, having new partner in last 12 months, male partner using condom in last 6 months, number of partners in last 6 months.

HPV, human papillomavirus; HR-HPV, High-risk HPV, defined as HPV types 16, 18, 31, 33, 35, 39, 45, 51, 52, 56, 58, 59, 66 (IARC 2007); STIs, sexually transmitted infections. 